Jean-Pierre Bastian, Kathy Rousselet, Françoise Champion (ed.), La Globalisation du religieux

Paris, L'Harmattan, 2001, 282 p.

\title{
Sébastien Fath
}

\section{(2) OpenEdition}

\section{Journals}

Édition électronique

URL : http://journals.openedition.org/assr/1592

DOI : $10.4000 /$ assr. 1592

ISSN : $1777-5825$

Éditeur

Éditions de l'EHESS

Édition imprimée

Date de publication : 1 avril 2002

Pagination : 87-151

ISBN : 2-222-96718-X

ISSN : 0335-5985

\section{Référence électronique}

Sébastien Fath, « Jean-Pierre Bastian, Kathy Rousselet, Françoise Champion (ed.), La Globalisation du religieux ", Archives de sciences sociales des religions [En ligne], 118 | avril - juin 2002, document 118.4, mis en ligne le 14 novembre 2005, consulté le 22 septembre 2020. URL : http:// journals.openedition.org/assr/1592; DOI : https://doi.org/10.4000/assr.1592 
On connaît la difficulté de cette méthode qui vise à éclairer tel fait ethnologique par un concept ou une élaboration psychanalytique. L'A. pratique celle-ci avec un relatif bonheur, même si le va-et-vient entre données de terrain et interprétation clinique ne s'avère pas toujours limpide pour le non-spécialiste. A.B., qui cite un corpus varié d'analystes, semble s'enraciner dans un freudisme classique: c'est l'impression qui ressort de la lecture des notes, car l'A. ne fournit pas de bibliographie.

Passons au versant anthropologique de cet ouvrage, que liront avec intérêt tous ceux qui cherchent à comprendre comment les sociétés transforment leurs membres en « êtres de culture ».

A.B., inspiré notamment par les travaux de M. Izard, voit à l'œuvre dans le groupe considéré une « dialectique structurante » qui oppose deux figures : «l'ancêtre» et le «sorcier». Chaque individualité les contient en puissance dès sa naissance : c'est au groupe d'affirmer la domination de l'instance ancestrale en la personne qui, ainsi, s'arrime peu à peu au corps social, grâce à ce long procès rituel par lequel le sujet est arraché à son animalité première et humanisé. Par contre, il faut refouler les «tendances sorcières ") dont chacun est porteur, parce que, si par malheur elles prennent le dessus, elles provoquent la «désocialisation » de l'individu, lequel bascule alors dans le monde nocturne des désirs irrépressibles et des pulsions dévastatrices. De ce fait, le tout premier traitement corporel que subit le sujet à son arrivée parmi les hommes - coupure du cordon, et surtout manipulation rituelle du placenta revêt une importance capitale, car c'est par lui que commence notre humanisation: le sorcier est celui auquel le corps social n'a pas réussi à infliger ces « morts symboliques » (ou « castrations ») ritualisées qui extirpent la part sorcière de l'individu et le socialisent.

Ainsi, le destin individuel oscille entre ces deux polarités extrêmes - l'ancêtre, le sorcier -, dont l'opposition anime aussi le jeu social (dans des pages intéressantes, l'A. décrit les modalités urbaines, contemporaines de la «sorcellerie »). Il suffit qu'un rituel échoue, qu'un « déchet» refoulé réapparaisse (par ex. un de ces placentas qu'on avait soigneusement enfouis) pour que les pulsions sauvages, asociales menacent le sujet comme la société.

Car, et c'est un des mérites de cet ouvrage de nous le rappeler, il n'y a rien de plus fragile que notre propre humanité, en proie à de terribles «tendances sorcières ».

Frank Noulin.
118.4

BASTIAN (Jean-Pierre), ROUSSELET (Kathy), CHAMPION (Françoise), ed.

\section{La Globalisation du religieux. Paris,} L'Harmattan, 2001, 282 p.

Cet ouvrage collectif rassemble les contributions de vingt et un auteurs au colloque de l'Association Française de Sciences Sociales des Religions (AFSR), tenu à Paris à l'IRESCO, les 5 et 6 février 2000. Il affronte l'incontournable question de la globalisation, et de son effet sur les religions. Jean-Pierre Bastian, Françoise Champion et Kathy Rousselet résument ainsi la perspective : « Le religieux est lui aussi transformé, de façon aussi intense que rapide, par ces changements spatio-temporels » (p. 9) que l'on a pris l'habitude de définir par les termes «mondialisation» ou «globalisation ». Les principaux effets de ce phénomène sont le passage de « l'international » au «transnational » (les logiques nouvelles mises en oeuvre ne dépendent plus de la politique des États), le développement de 《réseaux » structurés par la demande individuelle ou associative, la constitution de nouvelles « hybridités ». Elle entraîne à la fois une orientation plus individuelle, mais aussi de nouvelles logiques associatives: dans un monde devenu sans frontières, l'association élective, la communauté de croyants rassure. Les pentecôtismes (étudiés par Jean-Pierre Bastian, Marion Aubrée, Laënnec Hurbon et David Lehman) paraissent particulièrement en affinité avec ce mouvement de globalisation, tandis que d'autres Églises choisissent, soit des stratégies de repli identitaire (Église orthodoxe russe, étudiée par Kathy Rousselet), soit des dynamiques oecuméniques nouvelles (étudiées par Yves Bizeul), ou des recompositions transdénominationnelles, telles qu'on les observe notamment au sein du protestantisme évangélique, au travers, par exemple, du phénomène de plus en plus important des mega-churches (analysé par Danièle HervieuLéger et Jean-Paul Willaime). L'Europe, objet des regards croisés de Matthias König, Claire de Galembert, Martine Cohen et Solange Wydmusch, constitue un dossier particulièrement révélateur du dépassement des Étatsnations et des défis auxquels sont du coup confrontées les religions. Il n'est pas possible de rendre compte de la richesse de chaque exposé. On se limitera à en recommander la lecture, non seulement pour la richesse d'informations proposées, mais aussi pour les clarifications conceptuelles (échelles « internationale», «transnationale», « locale », « glocalisation »...) et les vertus méthodologiques de la comparaison, illustrées avec bonheur dans de nombreuses communications. 
On se bornera à esquisser, in fine, deux suggestions critiques, inspirées de certaines réflexions conclusives de Jim Beckford et de Bertrand Badie. Le second pose ainsi cette question: «L'un des effets les plus étonnants de l'abolition des distances n'est-il pas que les frontières entre le religieux, le politique, l'économique, le social sont de moins en moins nettes et de plus en plus discutables?»(p. 267). Derrière cette simple question, jetée en conclusion, s'avance un immense débat, très peu traité lors du colloque : celui de l'articulation finalement discutable entre globalisation et modernité. Suivant les analyses sociologiques classiques, la modernité s'est construite dans une logique de différenciation fonctionnelle des sphères. Or, si l'on suit l'hypothèse de Bertrand Badie, la globalisation pourrait bien, en euphémisant toutes les frontières, mettre en péril cette fameuse "différenciation des sphères » sur laquelle la modernité s'est appuyée depuis les Lumières. Ce qui permettrait de poser la question " religion et globalisation » dans des termes autrement plus cruciaux que la question, certes centrale, du degré d'adaptation des religions au phénomène de mondialisation. En d'autres termes, si l'on suivait toujours l'hypothèse de Badie, la globalisation pourrait ébranler les bases d'une modernité " différenciée » (où le politique, l'économique, le religieux... sont clairement séparés) et éventuellement redonner aux religions (à certaines religions ?) une part plus importante qu'elles ne l'ont eue ces dernières décennies dans la gestion des identités, dans la définition du lien social. Quand l'État national «moderne » se délite, quand les réseaux prennent le pas sur les institutions territorialisées, l'hypothèse d'un retour à une expression sociale plus extensive des religions peut-elle être écartée ? La question mériterait sans doute d'être examinée plus avant. Quant à la suggestion inspirée de Jim Beckford, elle est la suivante : évoquant Marc Gaborieau qui souligne la diffusion fort ancienne des grandes religions «dans d'immenses parties du monde », ou les « mouvements religieux américains » qui mettent en place, dès le $\mathrm{XIX}^{\mathrm{e}}$ siècle, «des réseaux internationaux aussi puissants que ceux des entreprises commerciales et industrielles contemporaines » (p. 274), on ne peut s'empêcher de penser, surtout si l'on fait profession d'historien, à la tradition transnationale séculaire de nombreuses religions, comme l'islam ou le christianisme. Une réalité d'évidence est la très large antériorité des dynamiques religieuses transnationales sur les dynamiques politiques transnationales. Durant des siècles, tandis que l'Europe était politiquement morcelée, la «globalisation » est passée par l'Église catholique médiévale, au travers du mythe porteur de la «chrétienté ». De la même manière, tandis qu'une partie du protestantisme s'est fortement territorialisée du $\mathrm{XVI}^{\mathrm{e}}$ au XVIII' siècle sur la base d'une « confessionnalisation » nationale et étatique, une autre sensibilité protestante, de type conversionniste et revivaliste, s'est au contraire exprimée sur un axe résolument transnational, déterritorialisé. Le «Grand Réveil » des années 1734-1744, nourri par des échanges transatlantiques très intenses, traduit à merveille ces dynamiques, sur lesquelles s'est appuyé ce que l'on appelle le protestantisme évangélique. Cet arrière-plan historique (que l'on aurait pu compléter par des exemples issus de l'islam, du judaïsme, du bouddhisme) invite, en poursuivant sur la lancée de Jim Beckford, à relativiser la problématique des stratégies d'adaptation des religions à la globalisation. Elle est certes importante, mais elle paraît avoir fait l'objet d'un traitement presqu'unilatéral, comme si les religions étaient surtout «structurées» par le contexte international, et non, également, « structurantes ». En d'autres termes, il eut sans doute été intéressant de renverser aussi la perspective, et de se demander en quoi les religions ont parfois préparé, contribué, annoncé la globalisation. A l'interrogation sur l'impact de la globalisation sur les religions répond donc celle (symétrique) de l'apport actif des religions au phénomène de globalisation. Effleurée parfois, cette question n'a pas fait l'objet du traitement qu'elle aurait sans doute mérité. Loin d'avoir toujours à s'adapter à la mondialisation (certaines religions fonctionnent depuis des siècles en réseaux transnationaux), on peut se demander si elles n'y ont pas directement contribué par leur influence culturelle et sociale. Est-ce un hasard si le pays aujourd'hui le plus favorable à l'abaissement des frontières douanières (les États-Unis) est aussi celui dont la culture religieuse majoritaire (fortement colorée de type « évangélique ») refuse la territorialisation des appartenances (modèle de la congrégation plus que de la paroisse) ? D'autres facteurs (économiques, culturels) interviennent évidemment aussi, mais la variable religieuse «structurante » peut-elle être ignorée pour autant ? Ces questions, loin de limiter l'intérêt de l'ouvrage recensé, lui rendent ainsi hommage. C'est grâce au brio des contributions, à la fécondité des analyses croisées, à l'audace des mises en perspectives introductives et conclusives qu'il est possible non seulement d'obtenir des réponses, mais aussi de poser des questions nouvelles.

Sébastien Fath. 